\title{
Distribuição da pressão estática em uma coluna de canola: análise por elementos finitos
}

\author{
Ednilton T. de Andrade ${ }^{1}$, Sandra M. Couto ${ }^{2}$ \& Daniel M. de Queiroz ${ }^{2}$ \\ 1 DEA/UFV, atualmente DCA/UFLA. CEP 37200-000, Lavras, MG. Fone: (35) 3821-5951. E-mail: ednilton@ufla.br (Foto) \\ 2 DEA/UFV. Viçosa, MG. E-mail: scouto@mail.ufv.br e queiroz@mail.ufv.br
}

Protocolo $064-13 / 06 / 2000$

\begin{abstract}
Resumo: A passagem de um fluido através de um leito fixo de partículas encontra-se presente nos procedimentos de aeração e de secagem de uma massa de grãos, usados para a conservação da qualidade do produto. Neste trabalho, usando-se a técnica de elementos finitos, modelou-se a queda de pressão estática em um leito recheado de grãos de canola Iciola-41 (Brassica napus L. var. olifera) submetido a diferentes fluxos de ar. Utilizando-se um processo de otimização, determinou-se a permeabilidade do leito aos diferentes fluxos de ar. Os resultados de queda de pressão utilizando-se a técnica de elementos finitos, quando comparados com resultados provenientes de alguns modelos clássicos, foram os que apresentaram melhor desempenho. 0 erro relativo, usando-se a técnica de elementos finitos, variou na faixa $0,7-24,3 \%$ e, para todas as simulações, o erro foi igual a 5,3\%.
\end{abstract}

Palavras-chave: queda de pressão, fluxo de ar, elementos finitos

\section{Static pressure distribution in a bed of rapeseed grains: Finite element analysis}

\begin{abstract}
The flow of a fluid through a porous medium occurs during the aeration and drying stages of a mass of grains, used for the product quality conservation. In this work, using the finite element technique, the variation of the static pressure in a bed of rapeseed Iciola-41 (Brassica napus L. var. olifera) submitted to different airflows was modeled. Through an optimization process, the permeability of the bed to the different airflows was also determined. The results of the finite element analysis, when compared with those obtained from some classical models, presented the best performance. The relative error using the finite element technique varied in the range $0.7-24.3 \%$ while the relative error for all simulations was approximately equal to $5.3 \%$.
\end{abstract}

Key words: pressure drop, airflow, finite-elements

\section{INTRODUÇ̃̃O}

Em engenharia agrícola, são comuns os procedimentos de aeração e de secagem de uma massa de grãos, usados para a conservação da qualidade do produto. Nesses procedimentos, o ar é forçado por um ventilador, a escoar através do material, considerado leito fixo de partículas. O sucesso desses processos depende da uniformidade da distribuição do ar no interior da massa de grãos que, por sua vez, depende da resistência que o produto oferece à passagem do fluido.

Teoricamente, o problema de escoamento de fluidos é definido pelas leis de conservação de massa, de momentum e de energia. Quando se consideram as seguintes restrições: (a) o fluido é Newtoniano, (b) o fluido é incompressível, (c) não ocorre mudança de fase, (d) o escoamento é isotérmico e permanente, (e) o domínio espacial do problema não muda com o tempo e (f) a viscosidade do fluido é constante, as equações de conservação podem ser expressas como:

Conservação de massa

$$
\nabla \vec{V}=\frac{\partial V_{x}}{\partial x}+\frac{\partial V_{y}}{\partial y}+\frac{\partial V_{z}}{\partial z}=0
$$

em que:

$\vec{V}=V_{x} \vec{i}+V_{y} \vec{j}+V_{z} \vec{k}$ - vetor velocidade do fluido com componentes $\mathrm{V}_{\mathrm{x}}, \mathrm{V}_{\mathrm{y}} \mathrm{e} \mathrm{V}_{\mathrm{z}}$ nas direções $\mathrm{x}, \mathrm{y}$ e $\mathrm{z}$, respectivamente $\vec{i}, \vec{j}$ e $\vec{k}$ - vetores unitários nas direções $x$, y e $z$, respectivamente

$\nabla=\frac{\partial}{\partial x} \vec{i}+\frac{\partial}{\partial y} \vec{j}+\frac{\partial}{\partial z} \vec{k}$ - operador vetorial 


\section{Equações de momentum}

$$
\begin{aligned}
& \rho\left(\frac{\partial\left(V_{x} V_{x}\right)}{\partial x}+\frac{\partial\left(V_{y} V_{x}\right)}{\partial y}+\frac{\partial\left(V_{z} V_{x}\right)}{\partial z}\right)= \\
& \rho g_{x}-\frac{\partial P}{\partial x}+R_{x}+\mu_{e}\left(\frac{\partial^{2} V_{x}}{\partial x^{2}}+\frac{\partial^{2} V_{x}}{\partial y^{2}} \frac{\partial^{2} V_{x}}{\partial z^{2}}\right) \\
& \rho\left(\frac{\partial\left(V_{x} V_{y}\right)}{\partial x}+\frac{\partial\left(V_{y} V_{y}\right)}{\partial y}+\frac{\partial\left(V_{z} V_{y}\right)}{\partial z}\right)= \\
& \rho g_{y}-\frac{\partial P}{\partial y}+R_{y}+\mu_{e}\left(\frac{\partial{ }^{2} V_{y}}{\partial x^{2}}+\frac{\partial^{2} V_{y}}{\partial y^{2}} \frac{\partial^{2} V_{y}}{\partial z^{2}}\right) \\
& \rho\left(\frac{\partial\left(V_{x} V_{z}\right)}{\partial x}+\frac{\partial\left(V_{y} V_{z}\right)}{\partial y}+\frac{\partial\left(V_{z} V_{z}\right)}{\partial z}\right)= \\
& \rho g_{z}-\frac{\partial P}{\partial z}+R_{z}+\mu_{e}\left(\frac{\partial^{2} V_{z}}{\partial x^{2}}+\frac{\partial^{2} V_{z}}{\partial y^{2}} \frac{\partial^{2} V_{z}}{\partial z^{2}}\right)
\end{aligned}
$$

em que:

P - pressão do fluido

$\mathrm{g}_{\mathrm{x}}, \mathrm{g}_{\mathrm{y}}$ e $\mathrm{g}_{\mathrm{z}}$ - componentes do vetor aceleração da gravidade $\left(\vec{g}=g_{x} \vec{i}+g_{y} \vec{i}+g_{z} \vec{i}\right)$ nas direções das coordenadas espaciais $\mathrm{x}, \mathrm{y}$ e $\mathrm{z}$, respectivamente

$\mathrm{R}_{\mathrm{x}}, \mathrm{R}_{\mathrm{y}}$ e $\mathrm{R}_{\mathrm{z}}$ - componentes de um vetor adicional qualquer $\left(\vec{R}=R_{x} \vec{i}+R_{y} \vec{i}+R_{z} \vec{i}\right)$ (fontes ou sorvedouros) nas direções $x, y$ e $z$, respectivamente

$\mu_{\mathrm{e}} \quad$ - viscosidade efetiva

$\rho \quad$ - densidade do fluido

O tratamento analítico do escoamento de fluidos em leitos de partículas é bastante complexo, enquanto as partículas que compõem o leito possuem, em geral, diferentes formas e tamanhos, o fluido escoa por complicados labirintos e a geometria do escoamento difere de maneira não previsível de uma região para outra do material. Por estas razões, a análise do escoamento de fluidos através de leitos de partículas tem sido feita de forma empírica. Na mecânica dos fluidos de meios porosos, as equações de conservação são substituídas por numerosas observações experimentais resumidas matematicamente, como a lei de Darcy (Bejan, 1984).

Um grande número de pesquisadores tem determinado experimentalmente a queda de pressão de um fluido ao longo de seu escoamento, através de leitos de partículas, sendo muitos desses leitos compostos de grãos agrícolas: Dentre esses, citam-se: Kay et al. (1989), milho; Sinicio et al. (1992), trigo; Alagusundaram \& Jayas (1990), grãos e oleaginosas; Jayas \& Sokhansanj (1989) canola com impurezas.

Santos (1996) propôs o seguinte modelo para expressar a queda de pressão estática por unidade de comprimento, $\nabla \mathrm{P}$, em uma coluna de canola em função do fluxo de ar, V, de propriedades do ar (viscosidade, $\mu$, e massa específica, $\rho$ ) e de características físicas da canola (diâmetro médio do grão, D, e porosidade da massa de grãos, $\varepsilon$ ):

$$
\nabla \mathrm{P}=180 \frac{\mu}{\mathrm{D}^{2}} \frac{(1-\varepsilon)^{2}}{\varepsilon^{3}} \mathrm{~V}+10,93 \frac{\rho}{\mathrm{D}} \frac{(1-\varepsilon)}{\varepsilon^{3 / 2}} \mathrm{~V}^{2}
$$

Usando os dados de seus experimentos, Santos (1996) determinou, por meio de regressão, os parâmetros empíricos (a, b, A, B, M e N) necessários à utilização de três modelos clássicos para expressar o gradiente de pressão em função do fluxo de ar imposto a uma coluna de canola: o de Shedd (1953):

$$
\nabla \mathrm{P}=\mathrm{a} \mathrm{V}^{\mathrm{b}}
$$

o de Hukill \& Ives (1955):

$$
\nabla \mathrm{P}=\frac{\mathrm{A} \mathrm{V^{2 }}}{\ln (1+\mathrm{BV})}
$$

e o de Hunter (1983):

$$
\nabla \mathrm{P}=\mathrm{MV}+\mathrm{NV}^{2}
$$

Técnicas numéricas têm sido amplamente usadas na resolução de problemas de escoamento de fluidos, dentre as quais a técnica de elementos finitos vem sendo usada com sucesso por vários pesquisadores, para a resolução de diferentes problemas envolvendo escoamentos. Em contraste com as soluções analíticas, que mostram o comportamento exato de um sistema em qualquer um dos seus pontos interiores, as soluções numéricas aproximam as soluções exatas somente em pontos discretos do sistema, denominados nós. No método dos elementos finitos, as etapas básicas consistem em (a) dividir a região sob investigação em elementos finitos, isto é, dividir a região em nós e elementos; (b) assumir uma função contínua de aproximação, função de forma, para representar a solução para cada elemento; (c) desenvolver equações para um elemento; matriz para o elemento e (d) gerar a solução completa pelo acoplamento ou conexão das soluções individuais para os elementos, construção da matriz de rigidez global, fazendo com que haja continuidade nos contornos inter-elementares.

No método dos elementos finitos, aplicado ao escoamento de fluidos, a região espacial em estudo é dividida em elementos e as Eqs. (2) (3) e (4) são discretizadas para cada elemento. O método de Garlekin (Segerlind, 1984) é usado na obtenção das matrizes para os elementos. A seguir, estas matrizes são agrupadas de forma adequada, em uma única matriz, global, e o sistema de equações resultante é resolvido para cada grau de liberdade, separadamente. As equações de momentum são usadas para gerar uma expressão para a velocidade, em termos de gradientes de pressão, que é usada na equação de conservação de massa, após ter sido integrada por partes.

O conhecimento da queda de pressão do ar ao escoar em leitos de grãos, é essencial no dimensionamento de sistemas eficientes de aeração e secagem. Vários modelos têm sido 
propostos para expressar a queda de pressão em função da profundidade em coluna de grãos. Os valores dos desvios entre os resultados provenientes da aplicação desses modelos e os valores experimentais de gradientes de pressão com a profundidade, são variáveis e dependem do produto e da faixa de fluxo de ar sob consideração. Técnicas numéricas aplicadas em conjunto com processos de otimização, poderiam ser uma alternativa razoável na modelagem do escoamento de ar em meios porosos.

Em vista do exposto, o presente trabalho teve por objetivo (a) modelar, usando-se a técnica de elementos finitos, a queda de pressão estática em um leito recheado de partículas submetido a diferentes fluxos de ar e (b) determinar, através de um processo de otimização, a permeabilidade do leito aos diferentes fluxos de ar.

\section{MATERIAL E MÉTODOS}

\section{Leito de partículas e fluxos de ar}

Nas simulações das quedas de pressão no interior de um leito de partículas, utilizou-se da geometria de um duto de seção transversal circular $(0,1525 \mathrm{~m}$ de raio) com a $1,00 \mathrm{~m}$ de comprimento, posicionado na vertical. $\mathrm{O}$ duto foi recheado com grãos de canola Iciola-41 (Brassica napus L. var. olifera) a um teor de umidade de 0,08 b.s. e os fluxos de ar impostos na entrada do leito, foram: 0,$00063 ; 0,00126 ; 0,00209 ; 0,00314$; 0,$00419 ; 0,00628 ; 0,00838 ; 0,01256 ; 0,01885 ; 0,02722 ; 0,03560$; 0,$04816 ; 0,06073 ; 0,07539 \mathrm{e} 0,09214 \mathrm{~m}^{3} \mathrm{~s}^{-1} \mathrm{~m}^{-2}$.

\section{Análise por elementos finitos}

A determinação do perfil de pressão e da velocidade em problemas de escoamento de fluidos em dutos sem partículas, é obtida de uma maneira bastante eficaz, quando se usa a técnica de elementos finitos assessorada por "softwares" interativos para análise de sistemas, como por exemplo o ANSYS, disponível comercialmente. Os parâmetros de entrada para os programas computacionais desenvolvidos nesta área são, basicamente, as propriedades do fluido, as condições de contorno em todas as superfícies, abertas ou fechadas, do sistema e o tipo de análise a ser realizada. As condições de contorno nas superfícies abertas podem ser velocidades, pressões ou combinações desses parâmetros; como resultado, têm-se as distribuições de velocidade e de pressão nos nós provenientes da discretização do sistema.

Quando se trata de escoamento em leitos de partículas, a análise requer que sejam realizadas algumas alterações no sistema sob investigação. Pode-se, por exemplo, determinar a distribuição de velocidade no leito introduzindo-se, no programa, valores, determinados experimentalmente, da queda de pressão. Uma outra alternativa é a de não se considerar o leito e analisar o escoamento de um outro fluido, diferente do ar, no duto; neste caso, as propriedades do fluido devem ser tais que proporcionem a distribuição de pressão esperada.

Neste trabalho, o sistema ANSYS, versão 5.3, foi utilizado para o desenvolvimento de um programa-fonte, para realizar uma análise tridimensional do escoamento de ar em uma coluna fixa de canola. O produto no interior da coluna foi considerado equivalente a uma resistência distribuída no interior do duto, ou seja, correspondente aos termos $\mathrm{R}_{\mathrm{x}}, \mathrm{R}_{\mathrm{y}}$ e $\mathrm{R}_{\mathrm{z}}$, nas Eqs. (2), (3) e (4). Desta forma, a queda de pressão no leito foi investigada sem se considerar a geometria do meio poroso. A resistência ao escoamento, modelada como uma resistência distribuída, pode ser devida a um, ou a uma combinação, dos seguintes fatores: uma perda localizada de pressão $(\mathrm{F})$, um fator de fricção (f) ou um fator $(\mathrm{C})$ relacionado à permeabilidade $(\mathrm{K})$ do meio $(\mathrm{C}=1 / \mathrm{K})$. Assim, o gradiente de pressão total, na direção $\mathrm{x}$, pode ser expresso por (ANSYS, 1996):

$$
\frac{\partial \mathrm{P}}{\partial \mathrm{x}_{\text {resistência }}}=-\left\{F \rho \mathrm{V}_{\mathrm{x}}|\mathrm{V}|+\frac{\mathrm{f}}{\mathrm{D}_{\mathrm{h}}} \rho \mathrm{V}_{\mathrm{x}}|\mathrm{V}|+\mathrm{C}_{\mathrm{x}} \mu \mathrm{V}\right\}
$$

onde $\mathrm{D}_{\mathrm{h}}$ é o diâmetro hidráulico. No presente trabalho, o meio foi considerado isotrópico $\left(\mathrm{C}_{\mathrm{x}}=\mathrm{C}_{\mathrm{y}}=\mathrm{C}_{\mathrm{z}}=\mathrm{C}\right)$ e a fricção nas paredes do duto (f) e as perdas localizadas de pressão (F) foram desprezadas.

O duto foi dividido em 1440 elementos do tipo FLUID142, com oito nós, resultando em 1927 nós ao final da discretização, como mostra a Figura 1. As entradas do programa-fonte foram as propriedades do ar (assumidas constantes), o valor de $\mathrm{C}$, mencionado no texto como RD1 (resistência distribuída) e as condições de contorno, que, pela estrutura do programa computacional, devem ser impostas para todas as superfícies, abertas ou fechadas, que delimitam o meio sendo investigado. Assim, foram usadas, como condições de contorno: (1) velocidades nulas $\left(\mathrm{V}_{1}\right)$, segundo os três eixos coordenados, na parede lateral interna do duto; (2) velocidade constante e uniformemente distribuída $\left(\mathrm{V}_{2}\right)$ na base do duto, e (3) pressão $\left(\mathrm{P}_{3}\right)$ igual a zero (referência) em todos os nós da extremidade superior do duto.

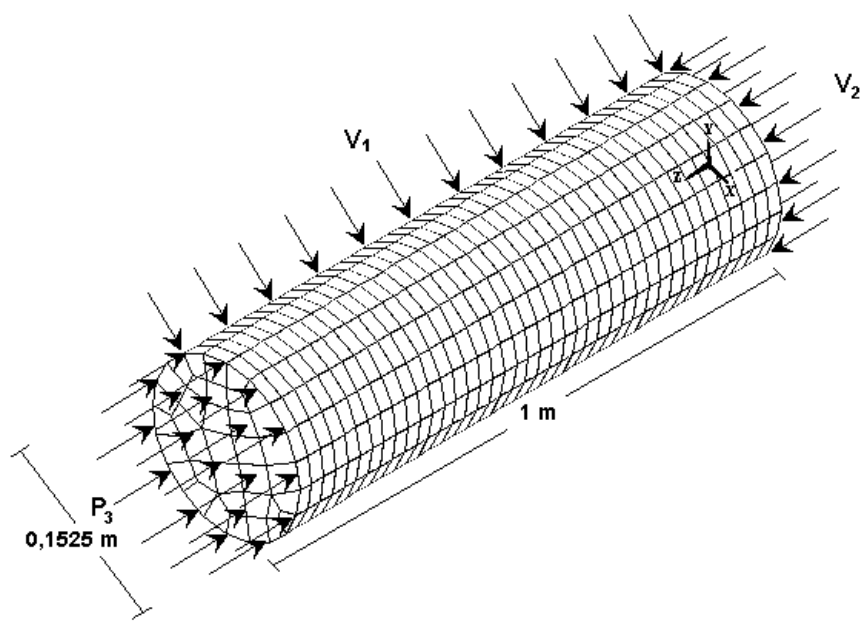

Figura 1. Malha e condições de contorno para análise por elementos finitos

\section{Otimização dos valores das resistências distribuídas}

Para a obtenção dos valores da resistência distribuída RD1, associada a cada velocidade de entrada do ar, foi utilizada uma das rotinas de otimização, do mesmo programa ANSYS, obedecendo-se os passos seguintes:

a) o programa-fonte era executado com valor inicial de resistência distribuída;

b) os valores de pressão em nós situados em determinadas profundidades ao longo do duto, eram obtidos; 
c) os erros relativos (erro $=100 \mathrm{x}\left|\mathrm{V}_{\mathrm{e}}-\mathrm{V}_{\mathrm{s}}\right| \mathrm{V}_{\mathrm{e}}$ ) entre os valores simulados, $\mathrm{V}_{\mathrm{s}}$, e os obtidos experimentalmente, $\mathrm{V}_{\mathrm{e}}$, para as mesmas profundidades, eram calculados;

d) determinava-se o somatório de todos os erros relativos, considerando-se todas profundidades, e esse resultado era armazenado em uma variável (SER) a ser minimizada ;

e) atribuíam-se valores, máximo e mínimo, para a variável RD1 e, também, o erro máximo admitido para a variável SER;

f) a rotina de otimização do programa ANSYS era acionada e determinava o valor de RD1 que conduzisse a um valor mínimo para a variável SER.

Nos ajustes das resistências distribuídas, determinadas pela técnica de elementos finitos, foram usados os valores experimentais (pressão estática versus profundidade) extraídos de Santos (1996).

\section{Comparação de modelos}

Os resultados da queda de pressão do ar escoando através de uma coluna de canola, determinados pela técnica de elementos finitos, foram comparados com aqueles provenientes dos modelos de Santos, Shedd, Hukill \& Ives e Hunter, Eqs. (5), (6), (7) e (8) respectivamente.

Os valores do diâmetro médio do grão e da porosidade da massa de canola usados na Eq. (5) foram iguais a 1,63 mm e $39,7 \%$, respectivamente (Santos, 1996). Os valores dos coeficientes dos três últimos modelos usados nas comparações, extraídos de Santos (1996) estão apresentados na Tabela 1.

\section{RESULTADOS E DISCUSSÃO}

Os resultados das simulações das variações de pressão em diferentes profundidades de uma coluna de canola, são apresentados na Figura 2, para fluxos de ar usados em processos de aeração e seca-aeração e na Figura 4, para fluxos de ar usados em secagens a temperaturas baixa e alta. Os erros relativos percentuais correspondentes, envolvidos nas simulações, são apresentados nas Figuras 3 e 5. Observa-se, nas Figuras 3 a 5, que os maiores erros relativos ocorreram para os valores de pressão simulados quando a profundidade na camada de canola era igual a $0,045 \mathrm{~m}$, ou seja, próximo à entrada de ar na coluna. Nas simulações foi considerada uma distribuição uniforme de velocidade do ar na entrada do duto mas, ao se observar a montagem experimental usada por Santos (1996) nota-se que, provavelmente, a condição de entrada do ar não corresponde à usada nas simulações.

Existem alguns problemas relacionados aos dados experimentais como, por exemplo, o da resolução da aparelhagem usada para a medição das pressões mais baixas e, também, o de valores de pressão que se desviam marcadamente da tendência dos outros valores experimentais como, por exemplo, o valor para a pressão a uma profundidade de $0,648 \mathrm{~m}$ (Figura 2).

A Tabela 2 apresenta os erros relativos médios envolvidos nas predições da queda de pressão na coluna de canola, submetida a diferentes fluxos de ar, usando-se os modelos de Hunter, Hukill e Ives e Shedd, para faixas geral e específica

Tabela 1. Valores dos coeficientes dos três últimos modelos usados nas comparações, extraídos de Santos (1996)

\begin{tabular}{|c|c|c|c|c|c|c|}
\hline \multirow{3}{*}{$\begin{array}{l}\text { Faixa de Fluxo } \\
\text { de } \operatorname{Ar}\left(\mathrm{m}^{3} \mathrm{~s}^{-1} \mathrm{~m}^{-2}\right)\end{array}$} & \multicolumn{6}{|c|}{ Modelo } \\
\hline & \multicolumn{2}{|c|}{ Shedd } & \multicolumn{2}{|c|}{ Hukill \& Ives } & \multicolumn{2}{|c|}{ Hunter } \\
\hline & $\mathrm{a}$ & $\mathrm{b}$ & A & B & $\mathrm{M}$ & $\mathrm{N}$ \\
\hline$(0,00063,0,01885)$ & 0,00022 & 0,89994 & 40144,58 & 5,79644 & 6156,8 & 72191,8 \\
\hline$(0,01885,0,09214)$ & 0,00024 & 0,89491 & 40099,17 & 5,82843 & 6953,5 & 17785,8 \\
\hline$(0,00063,0,09214)$ & 0,00023 & 0,90158 & 40143,76 & 5,83855 & 6915,8 & 18269,9 \\
\hline
\end{tabular}

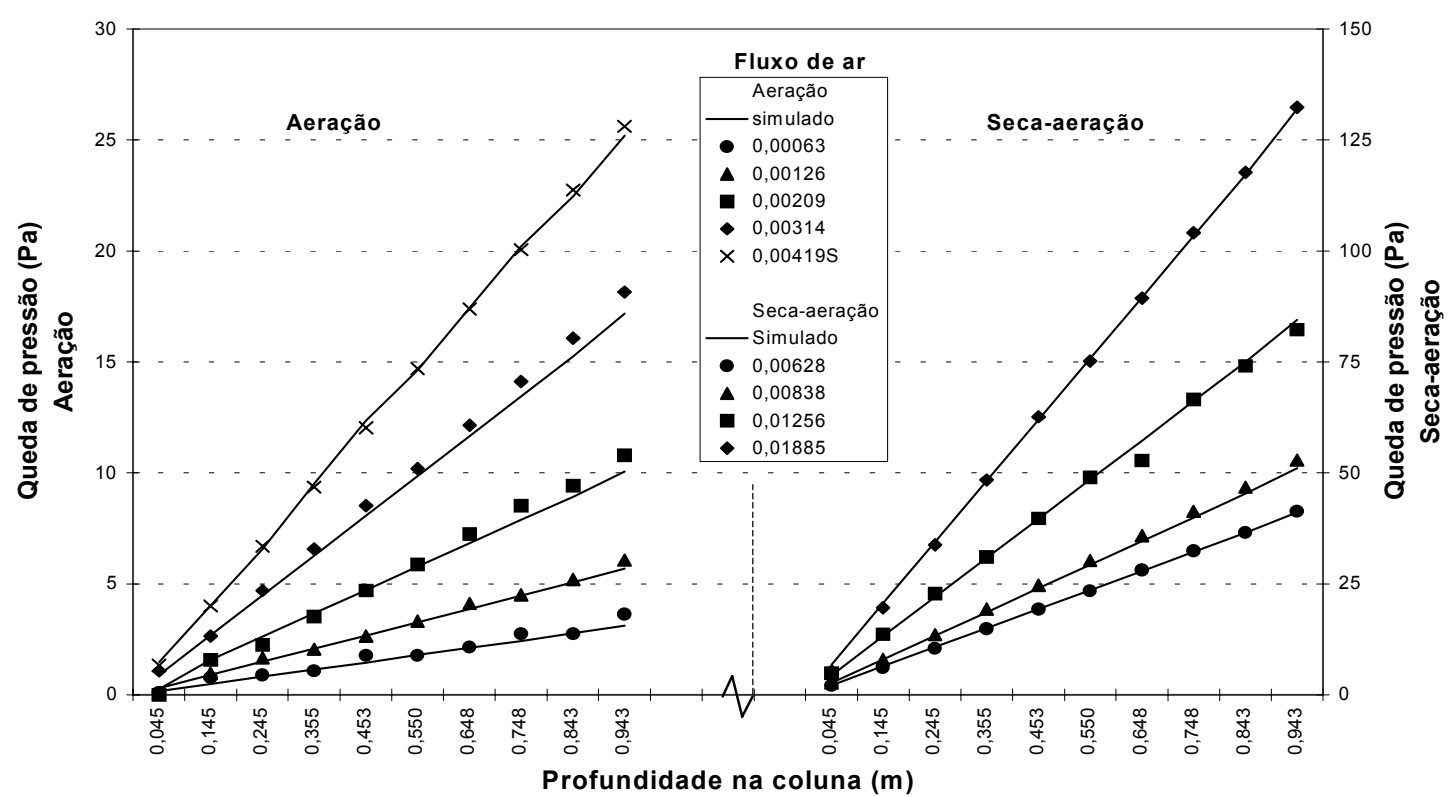

Figura 2. Queda de pressão em função da profundidade em uma coluna de canola, para fluxos de $\operatorname{ar}\left(\mathrm{m}^{3} \mathrm{~s}^{-1} \mathrm{~m}^{-2}\right)$ usados em sistemas de aeração e seca-aeração 


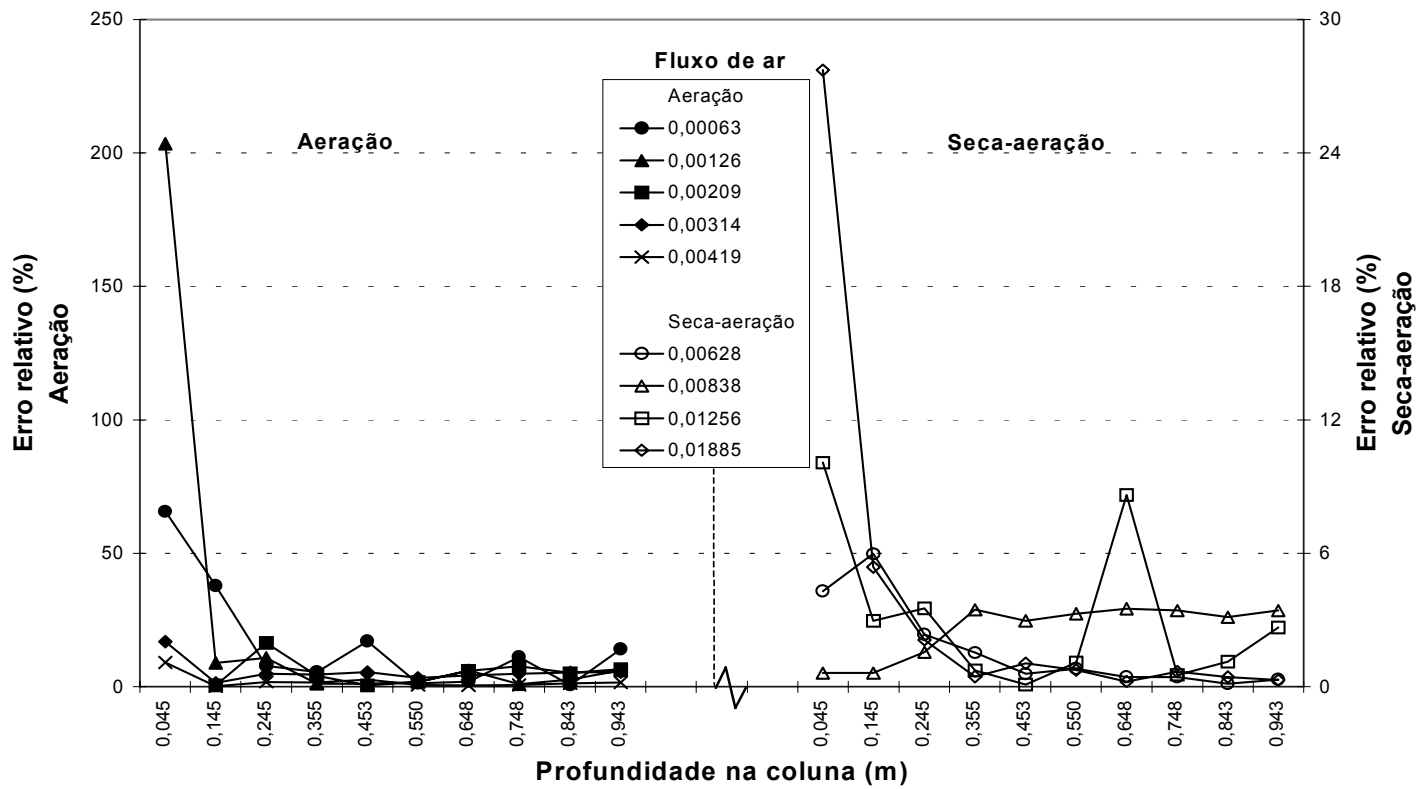

Figura 3. Erros relativos envolvidos nos valores simulados para a queda de pressão nas várias profundidades da coluna de canola, para fluxos de $\operatorname{ar}\left(\mathrm{m}^{3} \mathrm{~s}^{-1} \mathrm{~m}^{-2}\right)$ usados em sistemas de aeração e seca-aeração

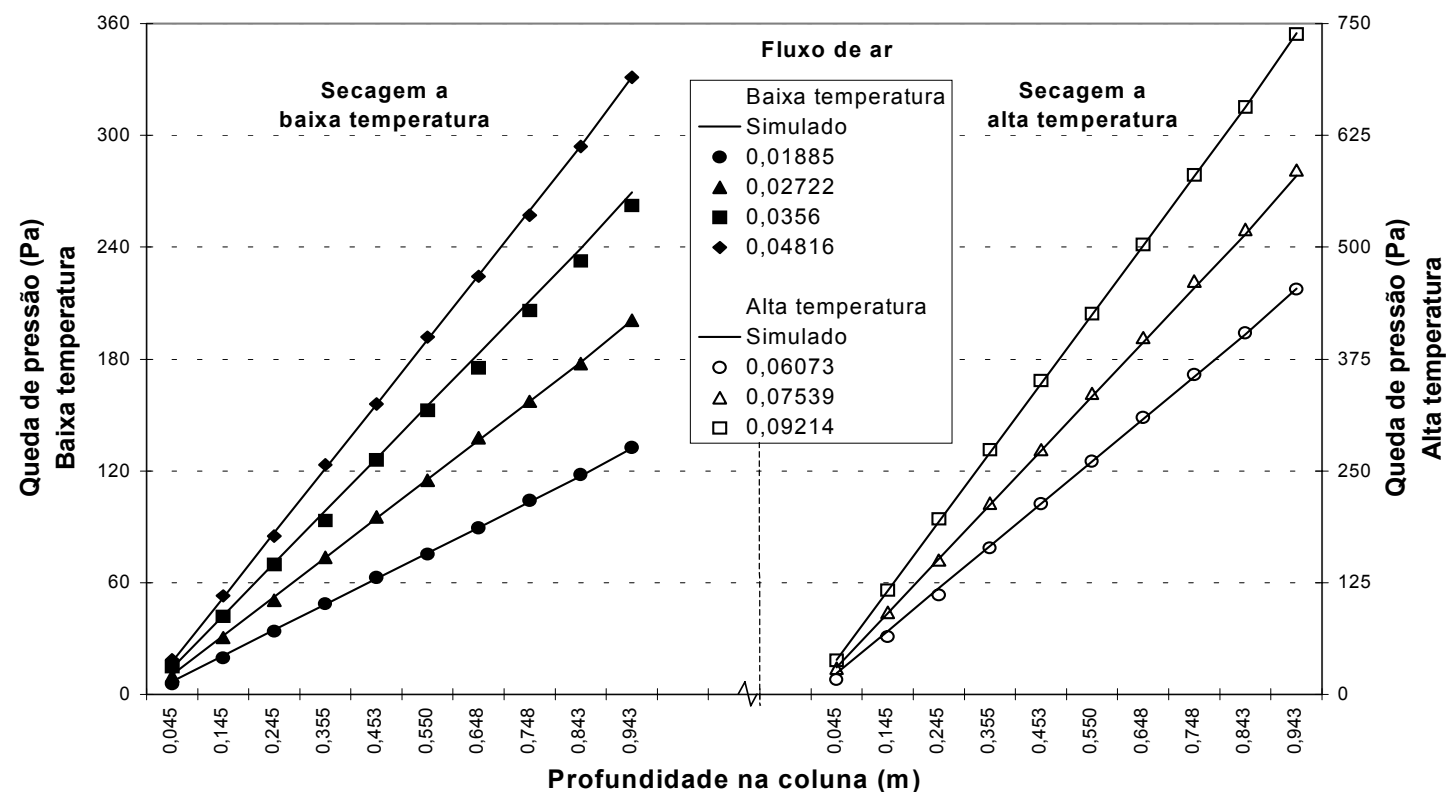

Figura 4. Queda de pressão em função da profundidade em uma coluna de canola, para fluxos de $\operatorname{ar}\left(\mathrm{m}^{3} \mathrm{~s}^{-1} \mathrm{~m}^{-2}\right) \mathrm{usados}$ em secagens a baixa e alta temperaturas

de fluxo de ar, o proposto por Santos (1996) e aqueles provenientes das simulações por elementos finitos, sob a denominação ANSYS. Para cada fluxo de ar esses erros representam a média dos erros determinados para cada uma das profundidades experimentais usadas por Santos (1996). Considerando-se uma média para todos os fluxos de ar investigados, os modelos de Hunter, Hukill e Shedd apresentaram erros relativos médios iguais a 9,9; 13,8 e 7,6\%, quando usados para faixas específicas de fluxo, e 13,7, 13,4 e $7,9 \%$, quando usados para uma faixa geral de fluxo de ar. Os modelos de Santos e aquele usando elementos finitos (ANSYS) apresentaram erros relativos médios iguais a 18,2 e 5,3\%, respectivamente. Observa-se que os três modelos que apresentaram menores erros relativos médios foram o ANSYS $(5,3 \%)$, o de Shedd, combinando faixas específicas de fluxo
$(7,6 \%)$ e o de Shedd, usando-se uma faixa geral $(7,9 \%)$ com erros variando nas faixas $[0,7,24,3],[1,5,32,3]$ e $[1,5,26,6]$ respectivamente. Observa-se que, sem dúvida, o modelo implementado usando o programa ANSYS, proporcionou as melhores estimativas. Dos quinze fluxos de ar testados, este modelo apresentou o menor erro em onze deles.

Considerando-se as faixas de fluxos de ar usados em sistemas de aeração, seca-aeração e secagem a temperaturas baixa e alta, a Figura 6 apresenta os valores (mínimo, médio e máximo) dos erros percentuais médios envolvidos na estimativa da queda de pressão na coluna de canola pelos modelos investigados. Observa-se que, para todos os modelos, os erros envolvidos na estimativa de queda de pressão na coluna submetida a fluxos de ar pequenos (sistemas de aeração) foram os maiores. 


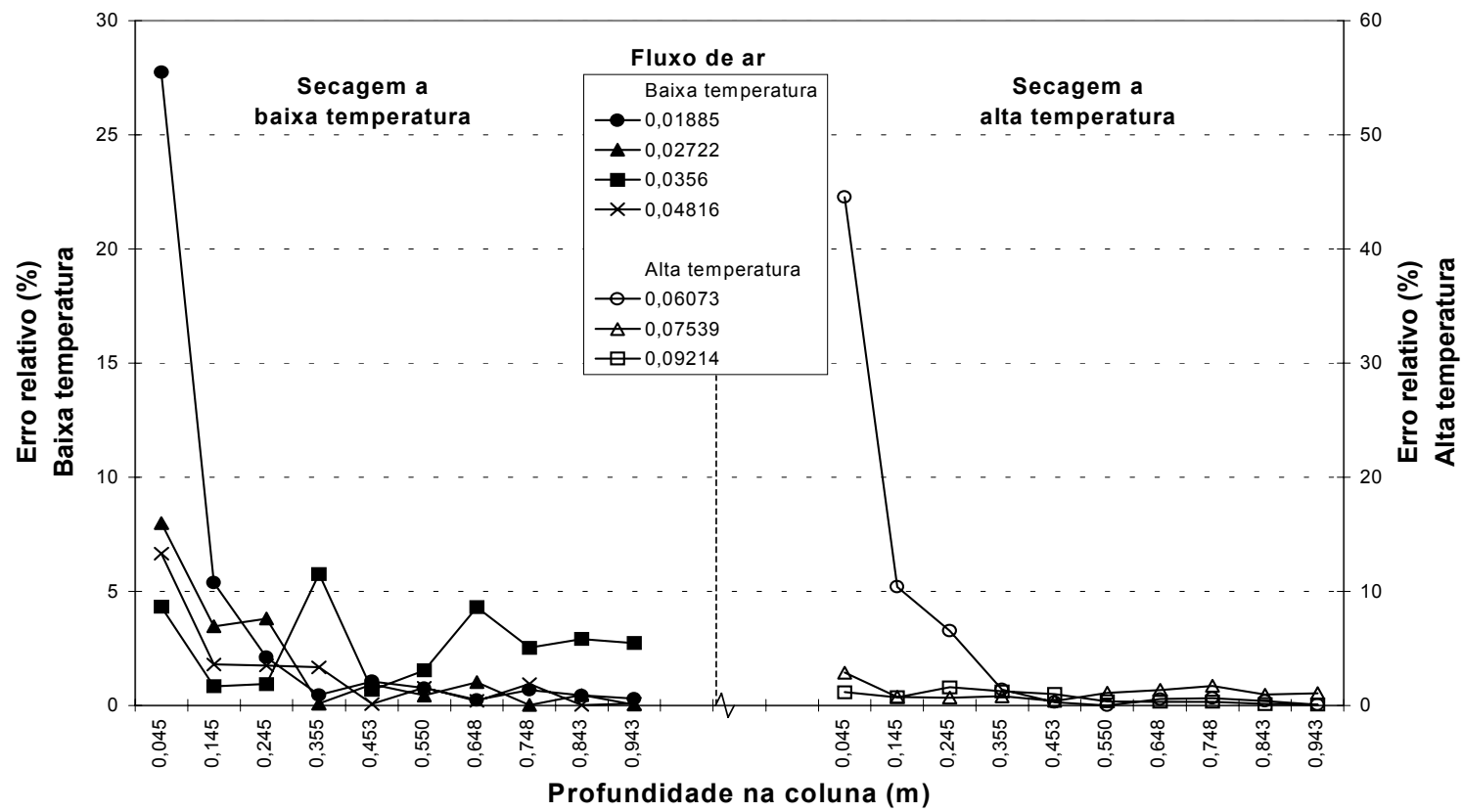

Figura 5. Erros relativos envolvidos nos valores simulados para a queda de pressão nas várias profundidades da coluna de canola, para fluxos de ar $\left(\mathrm{m}^{3} \mathrm{~s}^{-1} \mathrm{~m}^{-2}\right)$ usados em secagens a baixa e alta temperatura

Tabela 2. Erros relativos médios (\%) envolvidos na determinação da queda de pressão por meio de modelos em que foi utilizado os dados experimentais de Santos (1996)

\begin{tabular}{|c|c|c|c|c|c|c|c|c|c|c|c|c|c|c|c|}
\hline \multirow{2}{*}{ Modelo } & \multicolumn{15}{|c|}{ Fluxo de ar $\left(10^{-3} \mathrm{~m}^{3} \mathrm{~s}^{-1} \mathrm{~m}^{-2}\right)$} \\
\hline & 0,63 & 1,26 & 2,09 & 3,14 & 4,19 & 6,28 & 8,38 & 12,56 & 18,85 & 27,22 & 35,60 & 48,16 & 60,73 & 75,39 & 92,14 \\
\hline Hunter * & 21,2 & 49,8 & 23,8 & 7,6 & 2,8 & 3,6 & 2,9 & 4,4 & 4,3 & 3,8 & 4,5 & 7,9 & 7,2 & 2,2 & 2,3 \\
\hline Hunt & 33,0 & 66,3 & 36,5 & 15,6 & 8, & 3, & 6,8 & 4,8 & 2,4 & 4,1 & 4,7 & 7,7 & 7,1 & 2,2 & 2,3 \\
\hline Hukill * & 33,2 & 66,6 & 36,8 & 15,9 & 9,1 & 3,5 & 7,1 & 4,9 & 2,3 & 4,1 & 4,6 & 7,8 & 7,2 & 2,2 & 2,3 \\
\hline Hukill & 32,3 & 65,4 & 35,8 & 15,2 & 8,5 & 2,9 & 6,4 & 4,6 & 2,5 & 4,1 & 4,6 & 7,8 & 7,2 & 2,2 & 2,3 \\
\hline Shedd * & 15,6 & 32,3 & 14,6 & 3,9 & 3,6 & 3,8 & 3,5 & 4,6 & 3,4 & 6,0 & 5,4 & 7,4 & 6,5 & 1,5 & 2,1 \\
\hline Shedd & 17,7 & 26,6 & 8,6 & 5,5 & 8,8 & 9,1 & 2,8 & 7,0 & 4,6 & 5,3 & 4,9 & 7,7 & 6,6 & 1,5 & 2,2 \\
\hline Santos & 39,4 & 75,7 & 44,2 & 21,0 & 13,9 & 8,3 & 12,7 & 7,8 & 6,3 & 1,5 & 4,1 & 12,5 & 12,9 & 6,6 & 6,2 \\
\hline ANSYS & 16,4 & 24,3 & 5,4 & 5,7 & 2,0 & 1,7 & 2,6 & 3,2 & 3,9 & 1,8 & 2,7 & 1,4 & 6,5 & 1,2 & 0,7 \\
\hline
\end{tabular}

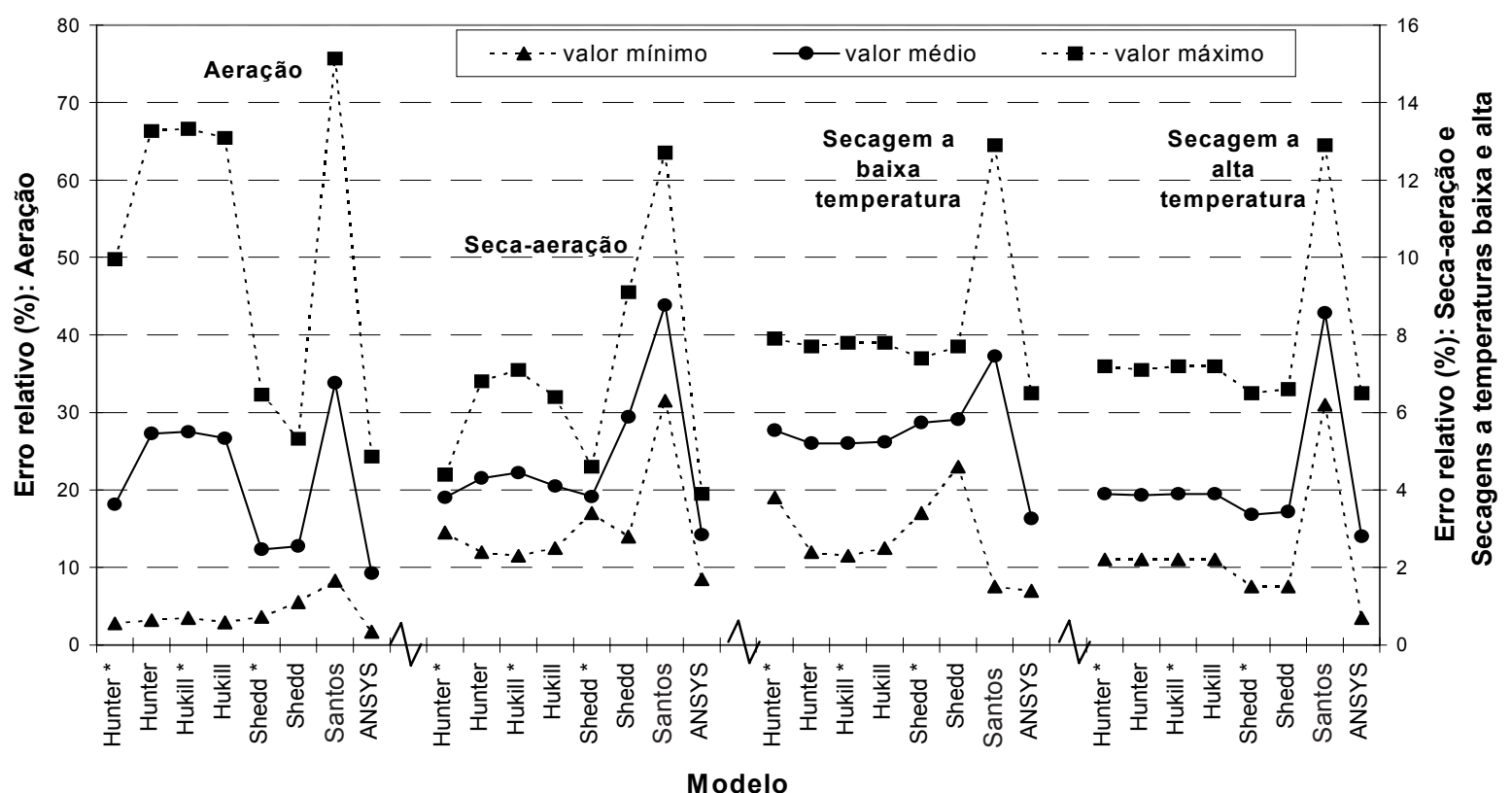

Figura 6. Erros relativos envolvidos nos modelos investigados para predizer o gradiente de pressão em uma coluna de canola submetida a fluxos de ar usados em sistemas de aeração $\left(0,00063\right.$ a $\left.0,00628 \mathrm{~m}^{3} \mathrm{~s}^{-1} \mathrm{~m}^{-2}\right)$, seca-aeração $\left(0,00628 \mathrm{a} 0,01885 \mathrm{~m}^{3} \mathrm{~s}^{-1} \mathrm{~m}^{-2}\right)$, secagem a temperaturas baixa $\left(0,01885\right.$ a $\left.0,06073 \mathrm{~m}^{3} \mathrm{~s}^{-1} \mathrm{~m}^{-2}\right)$ e alta $\left(0,06073\right.$ a $\left.0,09214 \mathrm{~m}^{3} \mathrm{~s}^{-1} \mathrm{~m}^{-2}\right)$ 


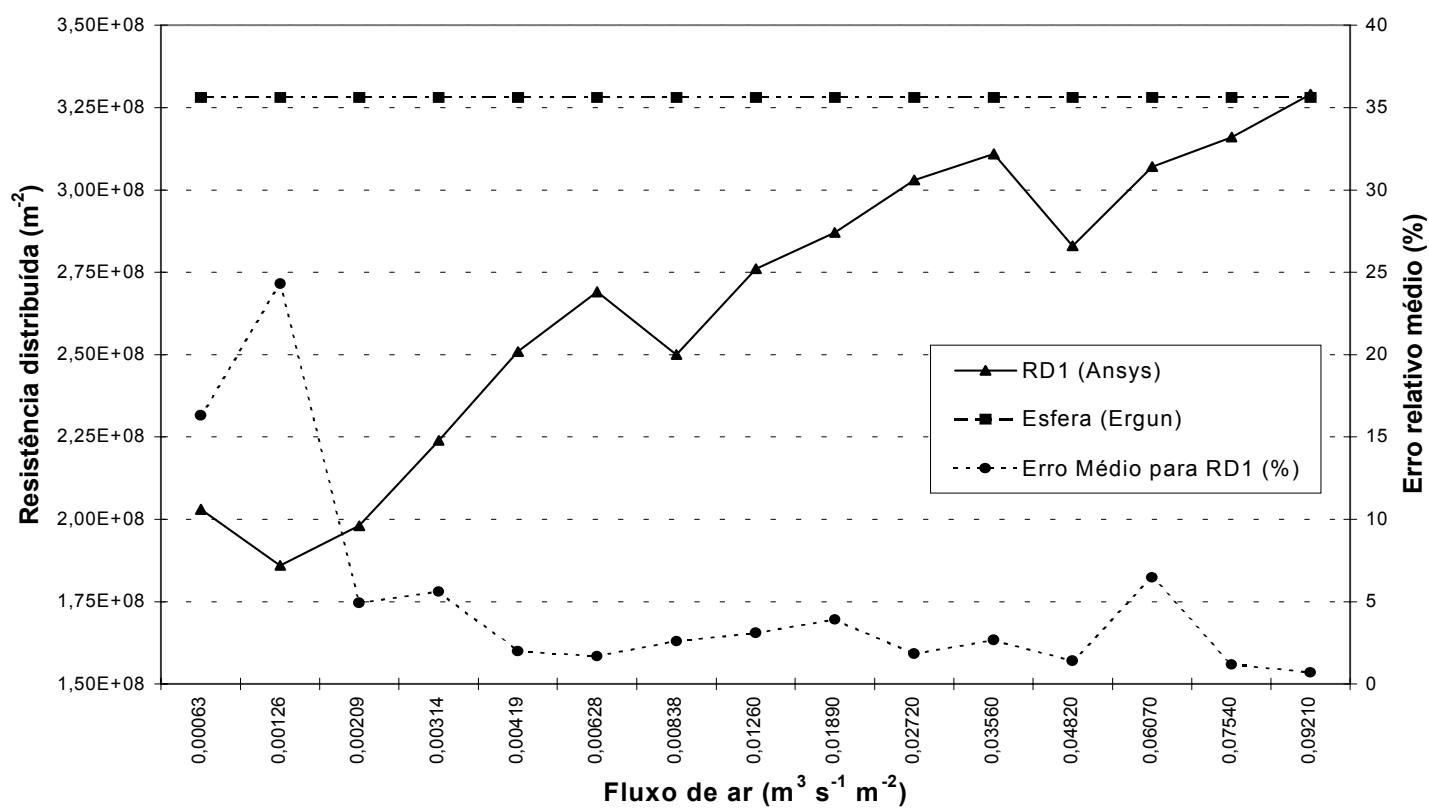

Figura 7. Valores da resistência distribuída (RD1) da coluna de canola, acompanhados dos erros da otimização, para diferentes fluxos de ar

Os valores das resistências distribuídas (RD1) representativas do leito de canola, para cada valor de fluxo de ar, provenientes do processo de otimização e acompanhados dos erros relativos envolvidos na otimização, são apresentados na Figura 7; esses valores variaram de $1,8578 \times 10^{8}$ a 3,2945 x $10^{8} \mathrm{~m}^{-2}$ e, considerando-se todos os fluxos de ar, tem-se uma resistência média igual a $(2,67 \pm 0,46) \times 10^{8} \mathrm{~m}^{-2}$.

Tentou-se manter os erros médios de simulação durante a otimização abaixo de 5\%, mas em alguns casos (fluxos de $0,00063,0,00126,0,00314$ e $0,06073 \mathrm{~m}^{3} \mathrm{~s}^{-1} \mathrm{~m}^{-2}$ ) não se obteve sucesso devido, provavelmente, a problemas nos dados experimentais. Apesar dos diferentes erros na otimização, observa-se (Figura 7) tendência dos valores da resistência distribuída aumentarem com acréscimos nos valores do fluxo de ar, o que pode ser expresso por uma equação do tipo $\mathrm{RD} 1=4 \times 10^{8} \mathrm{~V}^{-0,1104}\left(\mathrm{R}^{2}=0,889\right)$.

Sobre as considerações em que a análise foi realizada, a permeabilidade do leito (inverso da resistência distribuída) variou de $3,04 \times 10^{-9}$ a $5,38 \times 10^{-9} \mathrm{~m}^{2} \mathrm{e}$, numa média para todos os fluxos de ar, resultou em um valor igual a $(3,88 \pm 0,75) \times 10^{-9} \mathrm{~m}^{2}$. Ergun (1952) fornece uma expressão, Eq. (10), para a determinação da permeabilidade (considerada constante) de um leito recheado com esferas homogêneas.

$$
\mathrm{K}=\frac{\mathrm{D}^{2} \varepsilon^{3}}{150(1-\varepsilon)^{2}}
$$

A equação é válida para um Reynolds Modificado $\left(\mathrm{R}_{\mathrm{eM}}=\mathrm{Re} /(1-\varepsilon)\right)$ de até $4 \times 10^{3} \mathrm{e}$, como pode ser notado, a permeabilidade do leito é função apenas de propriedades geométricas. A resistência distribuída equivalente ao leito de canola, estimada através da Eq. (10) e tem valor igual a $3,28 \times 10^{8} \mathrm{~m}^{-2}$. Observa-se, da Figura 7, que a utilização da Eq. (10) só seria razoável para predizer a resistência de leitos submetidos a fluxos de ar mais altos; o melhor resultado ocorreu para um fluxo de $9,21 \times 10^{-2} \mathrm{~m}^{3} \mathrm{~s}^{-1} \mathrm{~m}^{-2}$.

\section{CONCLUSÕES}

Análises dos resultados obtidos neste trabalho permitiram as seguintes conclusões:

1. Todos os modelos investigados neste trabalho para se estimar a distribuição espacial de pressão estática em uma coluna de canola submetida a diferentes fluxos de ar, apresentaram altos erros para fluxos de ar usados em sistemas de aeração.

2. Considerando-se uma média para todos fluxos de ar investigados dos modelos testados, os que apresentaram menores erros relativos médios, 5,3, 7,6 e 7,9\%, foram, respectivamente, aqueles em que se usava o ANSYS (técnica de elementos finitos) de Shedd (combinando faixas específicas de fluxo) e o de Shedd (para uma faixa geral de fluxo de ar). Os erros envolvidos nas estimativas da queda de pressão por esses modelos variaram, respectivamente, nas faixas de $[0,7,24,3]$, $[1,5,32,3]$ e $[1,5,26,6]$.

3. A utilização do conceito da equivalência entre o leito de canola e uma resistência distribuída ao longo do duto, mostrou-se eficaz na modelagem, pelo método de elementos finitos, da queda de pressão em uma coluna de grãos de canola. Os valores das resistências distribuídas variaram de $1,8578 \times 10^{8}$ a $3,2945 \times 10^{8} \mathrm{~m}^{-2}$ e mostraram tendência de aumentarem com acréscimos nos valores do fluxo de ar. Considerando-se todos os fluxos de ar, tem-se uma resistência média igual a $(2,67 \pm 0,46) \times 10^{8} \mathrm{~m}^{-2}$.

4. A permeabilidade do leito de canola determinada a partir das simulações usando-se a técnica de elementos finitos, variou de $3,04 \times 10^{-9}$ a $5,38 \times 10^{-9} \mathrm{~m}^{2}$ e, numa média para todos os fluxos de ar, resultou em um valor igual a $(3,88 \pm 0,75) \times 10^{-9} \mathrm{~m}^{2}$.

5. A utilização da equação clássica de Ergun, usada para estimar a resistência ao fluxo de ar oferecida por uma coluna de partículas esféricas e homogêneas, só é razoável para predizer a resistência de leitos de canola submetidos aos fluxos de ar mais altos; o melhor resultado ocorreu para um fluxo de $9,21 \times 10^{-2} \mathrm{~m}^{3} \mathrm{~s}^{-1} \mathrm{~m}^{-2}$. 


\section{LITERATURA CITADA}

Alagusundaram, K.; Jayas, D.S. Airflow resistance of grains and oilseeds. Postharvest News and Information, Amsterdam, v.1, n. 4, p.279-283, 1990.

ANSYS. Ansys/Multiphysics Product (Program and Program Documentation), Realease 5.3, Houston: ANSYS Inc, 1996.

Bejan, A. Convection heat transfer. Durhan: Wiley Interscience, 1984. 477p.

Ergun, S. Fluid flow through packed columns. Chemical Engineering Progress, New York, v.48, p.89-94, 1952.

Hukill, W.V.; Ives, N.C. Radial air flow resistance of grains. Agricultural Engineering, St. Joseph, v.36, n.5, p.332-335, 1955.

Hunter, A.J. Pressure difference across an aeration seed bulk for some common duct and store cross-section. Journal Agricultural Engineering Research, Silsoe, v.28, n.5, p.437-450, 1983.
Jayas, D.S.; Sokhansanj, S. Design data on resistance of airflow through canola (Rapeseed). Transactions of the ASAE, St. Joseph, v.32, n.1, p.295-296, 1989.

Kay, R.L.; Bern, C.J.; Hurburgh Jr., C. R. Horizontal and vertical airflow resistance of shelled corn at various bulk densities. Transactions of the ASAE, St. Joseph, v.32, n.2, p.733-736, 1989.

Santos, V.P. Resistência ao fluxo de ar em uma coluna de grãos de canola Iciola-41 (Brassica napus L. var. olifera). Viçosa: Universidade Federal de Viçosa, 1996. 50p. Dissertação Mestrado

Segerlind, L.J. Applied finite element analysis. New York: John Wiley, 1984. 427p.

Shedd, C.K. Resistance of grain and seed to air flow. Agricultural Engineering, St. Joseph, v.34, n.9, p.616-619, 1953.

Sinicio, R.; Jayas, D.S.; Muir, W.E.; Sanderson, D.B. Finiteelement prediction of non-uniform airflow in fixed beds of wheat. Postharvest Biology and Technology, Amsterdam, v.2, p.51-59, 1992. 